\title{
0 ensino de inglês como língua estrangeira: desenvolvendo competência comunicativa no ensino regular
}

\section{Liliana Covino ${ }^{1}$}

\section{Resumo}

Em 2008, a autora teve a oportunidade de implementar um projeto em uma escola particular de Guarulhos, que tinha como objetivo ensinar inglês como Língua Estrangeira dentro da própria grade curricular da escola, desenvolvendo as quatro habilidades comunicativas, com ênfase na compreensão auditiva e fala. Tomou-se como referência o Common European Framework (CEF) para estabelecer quais habilidades poderiam ser alcançadas no período de cinco anos, e almejou-se atingir o nível de B1. Sendo assim, após um período de adaptações e ajustes, o desempenho dos alunos começou a ser medido . Este trabalho visa mostrar os resultados obtidos em 2012.

Palavras-chave: projeto, habilidade comunicativa, Inglês, Língua Estrangeira, CEF,

\begin{abstract}
In 2008, the author of this paper had the chance to implement a project in a private school in Guarulhos, which aimed teaching English as a foreign language to students as part of their regular school curriculum. Thus, students would have the chance to develop communicative competence and become fluent in the target language without the need of enrolling in a language school course after class. The Common European Framework (CEF) was used as a guide to establish what level students could reach. The students were challenged to reach the B1 level in a period of five years. After a period of time spent on adaptations, students' performance started to be measured. This paper presents the results of their performance in 2012.
\end{abstract}

Key words: Project, communicative competence, English, foreign language, CEF

${ }^{1}$ Mestre em Letras, Professora de Inglês da Instituição Eniac. 


\section{Introdução}

De acordo com os PCNs (1998, p. 19), a aprendizagem de uma Língua Estrangeira deve dar oportunidade ao aluno de se construir como sujeito do discurso e isso significa desenvolver sua capacidade de agir no mundo por meio da palavra em Língua Estrangeira, nas várias habilidades comunicativas.

Desta forma, o aluno começa a aprender uma língua a partir dos 11 anos e, ao concluir o ensino médio, terá estudado essa língua por sete anos. Este tempo deveria ser suficiente para tornar o aluno competente no domínio das habilidades oral, escrita, auditiva e leitura - da língua estudada. Mas isso não acontece no Brasil, e os próprios $\mathrm{PCNs}$ reconhecem este fato como uma fragilidade do ensino e sugerem, então, que se trabalhe pelo menos uma das habilidades:

Deve-se considerar também o fato de que as condições na sala de aula da maioria das escolas brasileiras (carga horária reduzida, classes superlotadas,pouco dominio das habilidades orais por parte da maioria dos professores,material didático reduzido a giz $e$ livro didático) podem inviabilizar o ensino das quatro habilidades comunicativas. Assim, o foco na leitura pode ser justificado pela função social das Linguas Estrangeiras no país e também pelos objetivos realizáveis tendo em vista as condições existentes. (PCN, 1998, p. 21)
Durante a elaboração de sua pesquisa e dissertação de mestrado intitulado "Os Parâmetros Curriculares Nacionais: sintonia entre teoria e prática?" a autora se deparou com a seguinte questão: se os alunos passam sete anos na escola, por quê não são fluentes em inglês?

Ao término da pesquisa, lendo os relatos dos alunos-futuros professores de inglês - a resposta estava evidente: o problema reside principalmente na falta de formação do professor, e uma carga horária reduzida.

Vera Lúcia Menezes de Oliveira e PAIVA em seu artigo "Memórias de aprendizagem de professores de Língua Inglesa" discorre sobre o problema da formação dos professores de inglês:

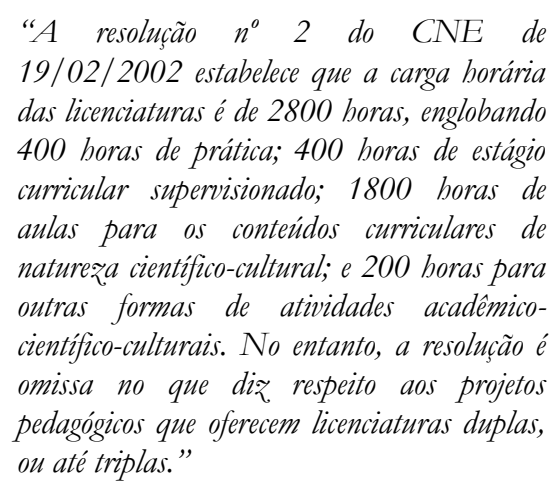

O grande problema enfrentado pelos cursos de licenciatura em língua estrangeira (LE) é o de que esses cursos estão, na maioria dos casos, atrelados às licenciaturas em língua portuguesa cujos conteúdos ocupam a maior parte da grade curricular. Como a legislação permite que as 
licenciaturas tenham a duração mínima de três anos, os empresários da educação alegam questões de mercado e de concorrência para empacotar a formação de professores em duas línguas, dentro de um período de três anos, sem nenhuma preocupação com o desenvolvimento da competência comunicativa do professor, para mencionar apenas uma das competências esperadas de um bom professor."

O que acontece nas salas de aula reflete a nossa triste realidade. A baixa desenvoltura dos brasileiros foi comprovada pelo EPI 2013 - Índice de Proficiência em Inglês, realizado pela EF Education First, escola especializada no ensino de idiomas e intercâmbios, que avaliou a gramática, vocabulário, leitura e compreensão de 1,7 milhão de adultos de 54 países.

O Brasil figurou na $38^{a}$ posição do ranking com uma avaliação de proficiência baixa. Entretanto, não é objeto deste trabalho discursar sobre os motivos que fazem com que o Brasil ainda não seja bilíngue. A questão que intrigou a autora foi a questão do tempo: sete anos na escola e não se adquiri nenhuma competência linguística em língua inglesa?

A autora, então, sentiu-se motivada a experimentar na prática: encontrar uma escola onde pudesse colocar seus conhecimentos referentes ao ensino de inglês como língua estrangeira. A oportunidade surgiu em 2008. O proprietário de um colégio particular em Guarulhos, já reconhecendo da necessidade do inglês no mercado de trabalho resolveu implementar na grade curricular o inglês como língua estrangeira. Muitas escolas oferecem um curso de inglês extracurricular.: o aluno deve frequentar as aulas no contra período com uma carga horária variada. Neste caso, a proposta foi inserir o ensino do idioma na própria grade curricular para que o aluno não tivesse necessidade de permanecer mais tempo na escola e adquirisse competência comunicativa no idioma. Buscou-se em Larsen-Freeman, a definição de competência comunicativa:

\begin{abstract}
Communicative competence involves being able to use te language appropriate to a given social context. To do this students need knowledge of the linguistic forms, meanings and functions. They need to know that many different forms can be used to perform a function and also that a single form can often serve a variety of functions. They must be able to choose from among these the most appropriate form, given the social context and the roles of the interlocutors They must also be able to manage the process of negotiating meaning with their interlocutors. L ARSENFREEMAN, 1986, p. 131)2.
\end{abstract}

Começou então um processo de implementação que sofreu muitas mudanças

\footnotetext{
2 Ter competência comunicativa significa ser capaz de usar a linguagem apropriada dentro de um dado contexto social. Para isso, os alunos precisam conhecer as estruturas linguísticas, seus significados e funções. Também precisam saber que muitas estruturas podem ser usadas para expressar a mesma função e que uma simples estrutura pode ser utilizada para expressar várias funções. Também devem ser capazes de escolher as estruturas e formas apropriadas para se expressarem de acordo com o contexto no qual estão inseridos: formal, informal etc. Também precisam ser capazes de dominar o processo de negociação de significado com seus interlocutores.( Tradução nossa)
} 
no decorrer de todos estes anos e que finalmente começou a ser medido em 2012.

Objetivo

Este relato tem como objetivo apresentar os resultados deste projeto que está em andamento desde 2008 e mostrará os resultados finais em 2014. A equipe de inglês tem como objetivo ensinar inglês ensinado as quatro habilidades: compreensão auditiva, fala, leitura e escrita para que os alunos desenvolvam competência comunicativa no idioma.

A equipe de professores foi cuidadosamente selecionada e atualmente é formada por oito professores, todos formados em Língua e Literatura Inglesas, com experiência em lecionar em cursos de idiomas. Os livros adotados foram desenvolvidos especialmente para ajudar a desenvolver as quatro habilidades.

Este projeto está sendo aplicado nas turmas da manhã do colégio do $6^{\circ}$ ano (fundamental II) ao $2^{\circ}$ ano do ensino médio e também nos 3 anos do ensino médio técnico.

Para as $6^{a}$ e $7^{a}$ séries do ensino fundamental foram escolhidos materiais da Oxford University Press, da coleção Project. Para as demais séries usamos o material da Cambridge University Press, da coleção Interchange.
Vale ressaltar aqui a carga horária total da disciplina de inglês:

Para quem começa no $6^{\circ}$ ano no colégio, ao terminar o ensino médio terá tido aproximadamente 321 horas de inglês (32 semanas -2 aulas de 50' $=53,5$ horas/ano). Para quem começa no médio técnico, após 3 anos terá tido aproximadamente 192 horas de inglês $(16$ aulas de 2 horas $=$ $32 /$ semestre $=64$ horas $/$ ano $)$

Este projeto tem a intenção de fazer com que um aluno que entre no colégio no $\sigma^{a}$ ano e faça todos os módulos do curso, sem necessidade de repetição, no final do ensino médio, após sete anos na escola, tenha atingido um nível de inglês B1.

Se faz necessário definir o significado do nível.B1

O Common European Framework of Reference for Languages é o documento utilizado para descrever o desempenho dos aprendizes de línguas estrangeiras na Europa. Elaborado pelo Conselho Europeu, foi considerado o principal item do projeto "Language Learning for European Citizenship" entre os anos de 1989 e 1996.

Seu principal objetivo é o de fornecer método de avaliação e ensino que se aplique a todos os idiomas dos países membros da Comunidade Européia. Em 2001, uma Resolução do Conselho Europeu recomendou o uso do CEFR para regulamentar os sistemas de validação das habilidades linguísticas. Desta forma, os seus 
seis níveis de referência se tornaram amplamente aceitos como padrão para nivelar o grau de conhecimento que uma pessoa possui de outra língua.

Devido à seriedade e comprometimento científico do projeto, muitas instituições, empresas e ONGs no mundo todo têm adotado o CEFRL como padrão na definição de níveis dos cursos de idiomas por eles oferecidos. Estes órgãos, porém, não trocaram o nome dos níveis por eles adotados. Eles apenas estabeleceram a correspondência entre seus níveis (elementar, básico, pré-intermediário, intermediário, avançado, etc) com os níveis do CEFRL, que são:

- A (Basic User - Usuário Básico)

- A1 - Breakthrough

Apresentar-se e fazer o mesmo com outras pessoas.

Fazer e responder a perguntas pessoais simples, como: onde mora, o que faz, o que gosta

\section{- $\mathrm{A} 2$}

Waystageaproximadamen te $180-200$ horas de aprendizado.

Dar informações pessoais básicas sobre a sua família, seu trabalho, estudo.Fazer compras, trocar informações diretas sobre assuntos rotineiros conhecidos, falar, de forma simples, de sua formação profissional e educacional, falar da sua cidade, empresa, país, de forma simples.
- B (IndependentUser Usuário Independente)

- B1Threshold (aproximadamente 350 a 400 horas de curso)

Lidar com situações de viagem em um país estrangeiro.

Escrever textos simples de assuntos conhecidos e de seu interesse.

Descrever experiências, ambições, situações.

Justificar, de forma simples, as suas opiniões

- B2Vantage (aproximadamente 500 a 600 horas de curso)

Compreender aspectos concretos e abstratos de um texto, mesmo que sejam assuntos técnicos em sua área acadêmica ou profissional.

Consegue interagir com falantes nativos sem muitos problemas.

Tem condições de falar sobre vantagens e desvantagens de um tópico.

Produzir textos mais detalhados sobre vários assuntos.

- C (ProficientUser Usuário Proficiente)

- C1EffectiveOperatio nalProficiency (aproximadamente 700 a 800 horas) 
Interpretar textos mais longos e complexos, mesmo que tenham um sentido implícito.

Expressar-se claramente, sem procurar palavras ou expressões.

Usar o idioma facilmente para situações sociais, acadêmicas, diárias e profissionais.

Escrever textos claros, detalhados sobre temas complexos

- C2Mastery(aproximadam ente 1000 a 1200)

Compreender quase tudo o que ouve e lê.

Condensar informações, fazer relatos

e reconstruir textos de forma eficiente.

Falar e se fazer entender de forma eficiente, fluente e precisa.

Para facilitar a compreensão dos resultados, foi feita a correlação entre livro usado com o nível atingido e a carga horária. Não é possível dizer exatamente quantas horas de estudo são necessárias para cada nível, pois isso varia muito de pessoa para pessoa; envolve motivação, estudo fora de sala de aula, contato com o idioma fora da sala de aula e estilo de aprendizagem do aluno. Sendo assim, a quantidade de horas deve ser considerada como referência.

Como se observa na descrição, para que um aluno consiga desenvolver competências e habilidades linguísticas em um nível B 1, ele precisa aproximadamente 300 horas de estudo. Infelizmente, mesmo em um período de sete anos na escola, a carga horária não atinge este tempo, pois apesar da contagem perfazer um total de 321 horas, não foram descontados dias de provas, eventos e tempo despendido com troca de salas de aula. Para os alunos que passam somente três anos na escola, então a carga horária é a metade. Porém, será feito de tudo para que o objetivo de B1 seja alcançado.

O material usado no fundamental 2 tem como objetivo encaixar o aluno no livro Inter 1B ao término do 9 ano (Nível A2 do CEF). Porém, isso depende da maturidade e desenvolvimento do aluno. Caso ele não consiga, será encaixado no nível em que se encontra.

Como pode se observar no quadro abaixo, entre o nível de A2 e B1 o aluno poderá prestar alguns testes de certificação internacional. Vide quadro abaixo. 


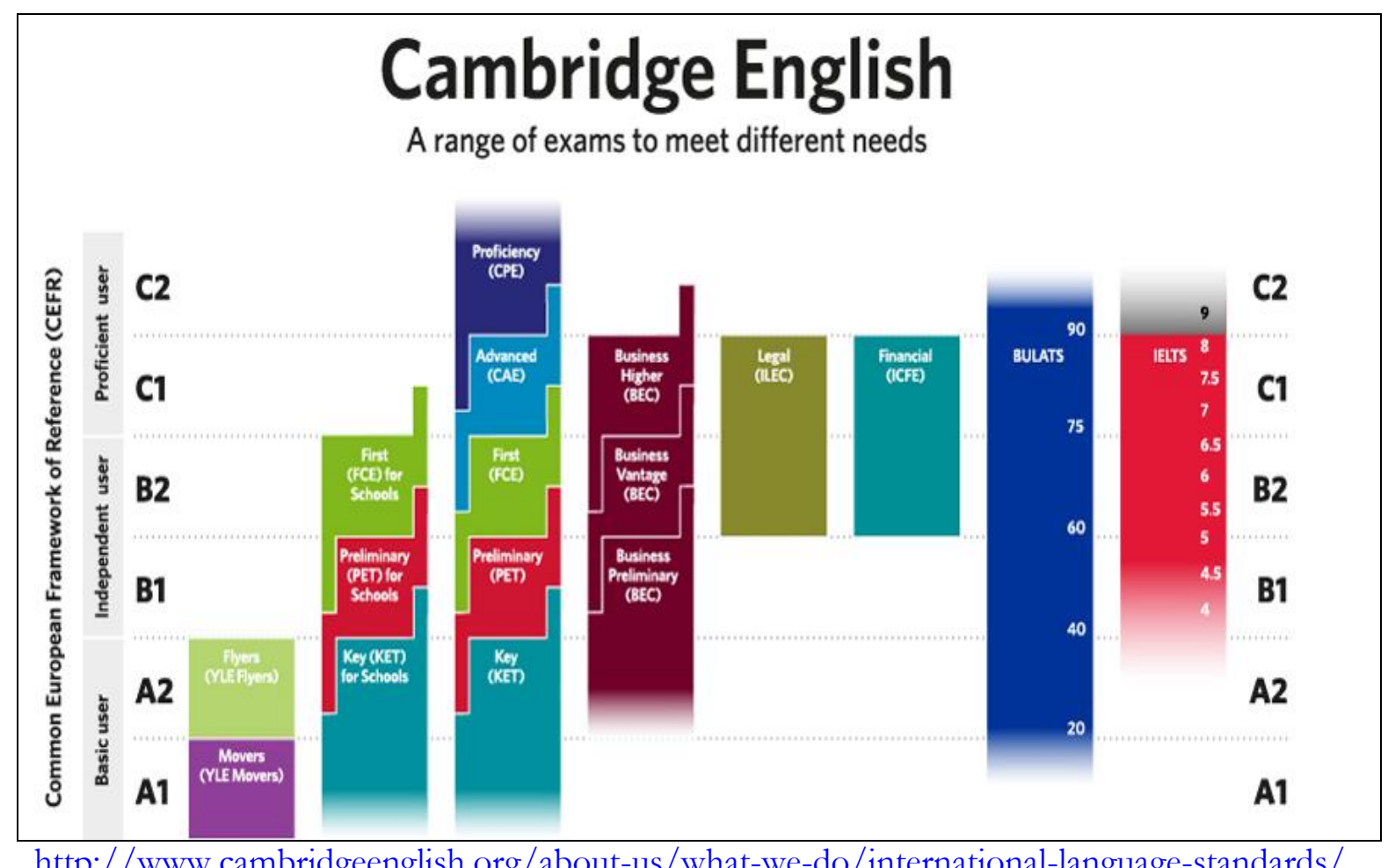

\section{Metodologia}

Para medir os níveis de aprendizagem dos alunos utilizou-se um teste cedido pela Cambridge University Press, editora que fornece os livros e o suporte pedagógico. Este teste tem ao todo 70 questões: 20 questões de compreensão auditiva e 50 de leitura e gramática. Este teste foi aplicado nos 9 anos e nos 3 anos do ensino médio regular, pois a aquisição de determinadas habilidades somente conseguem ser medidas após um determinado período de tempo. 137 alunos participaram desta avaliação. Como na época de realização do exame outubro de 2012 o portal virtual ainda não possuía uma ferramenta que pudesse ser usada para aplicação de provas, esses alunos fizeram o teste em papel. Eles foram levados ao laboratório de informática, e cada aluno acessou seu portal, abriu a prova e anotou as respostas na folha de respostas. Em uma aula foi realizado o teste de compreensão auditiva e em outra aula o outro teste com 50 questões.

No ensino médio técnico (do 1 ao 3) a prova foi feita eletronicamente pelo portal técnico. As questões foram inseridas e cada turma fez sua prova no próprio horário de aula de inglês.

Nos quadros seguintes mostraremos o gráfico com a quantidade de alunos, livro em que se encaixa e o respectivo nível do CEF. As turmas dos $9^{\circ}$. anos, são as que começaram o projeto em 2008 e pelos resultados podemos perceber que o $9^{\circ}$. ano encontra-se no nível A2. 


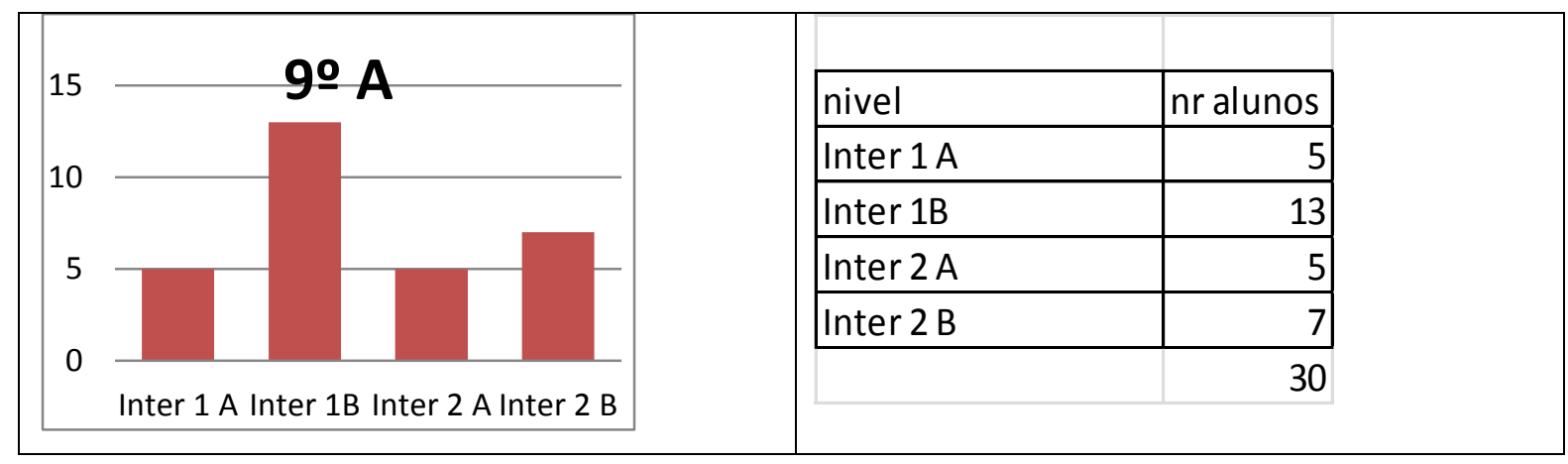

No gráfico abaixo pode-se perceber a referencia de livro e nível.

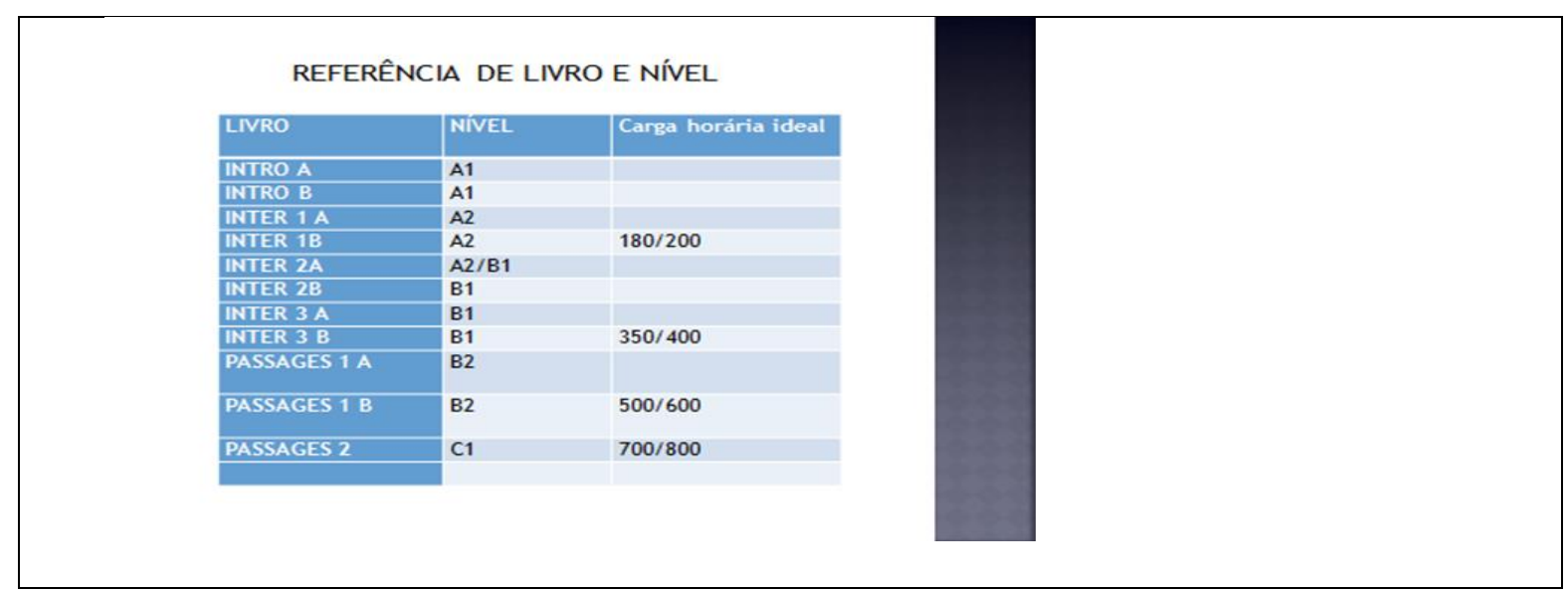

O mesmo se observa no $9^{\circ}$. B:

\begin{tabular}{|c|c|c|c|}
\hline \multirow{10}{*}{ 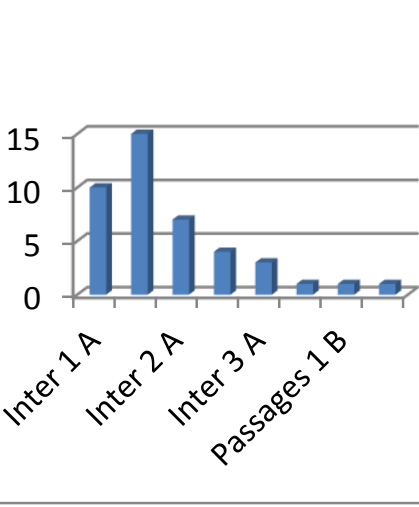 } & \multirow{10}{*}{$\begin{array}{r}90 \text { B } \\
\text { nr alunos }\end{array}$} & nivel & $\mathrm{nr}$ alunos \\
\hline & & Inter 1 A & 10 \\
\hline & & Inter $1 \mathrm{~B}$ & 15 \\
\hline & & Inter $2 \mathrm{~A}$ & 7 \\
\hline & & Inter 2 B & 4 \\
\hline & & Inter $3 \mathrm{~A}$ & 3 \\
\hline & & Inter 3 B & 1 \\
\hline & & \begin{tabular}{|l|} 
Passages $1 \mathrm{~B}$ \\
\end{tabular} & 1 \\
\hline & & Intro B & 1 \\
\hline & & & 42 \\
\hline
\end{tabular}

Nos terceiros anos do ensino médio regular o nível dos alunos se encontra entre B1.

No gráfico abaixo pode-se perceber o $3^{\circ}$.A.

8 Covino, L: 0 Ensino De Inglês Como Língua Estrangeira: Desenvolvendo Competência Comunicativa No Ensino Regular 


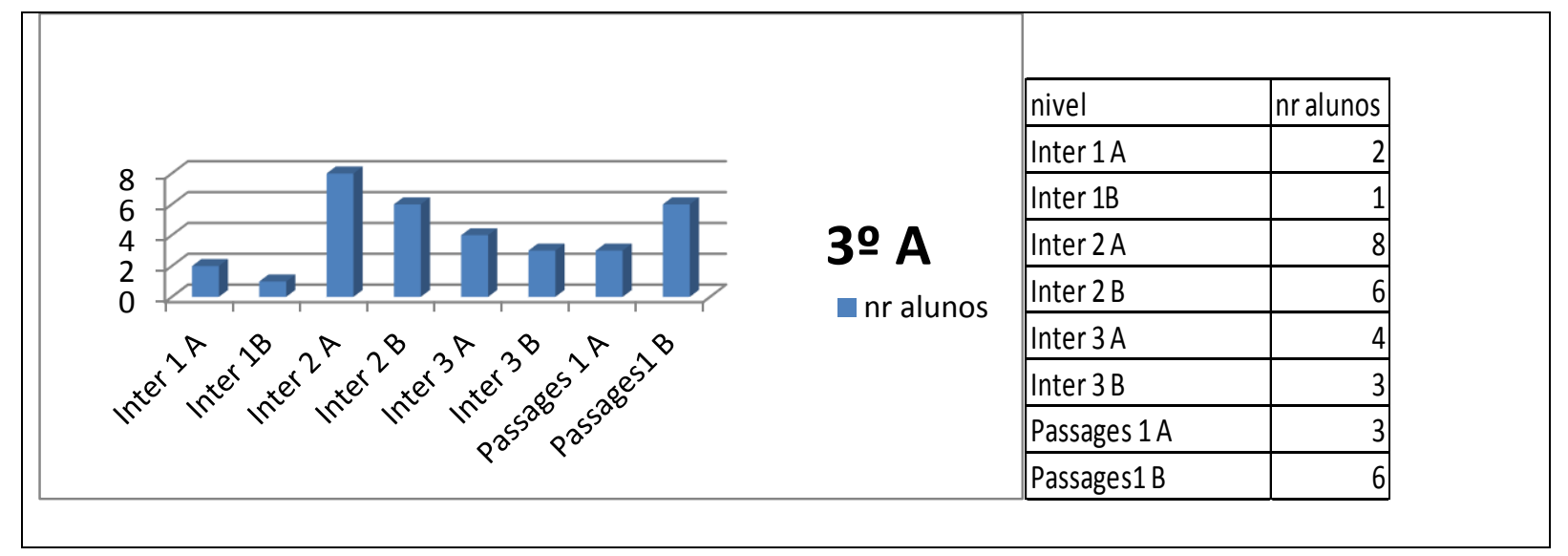

No gráfico abaixo pode-se perceber o $3^{\circ}$.B.

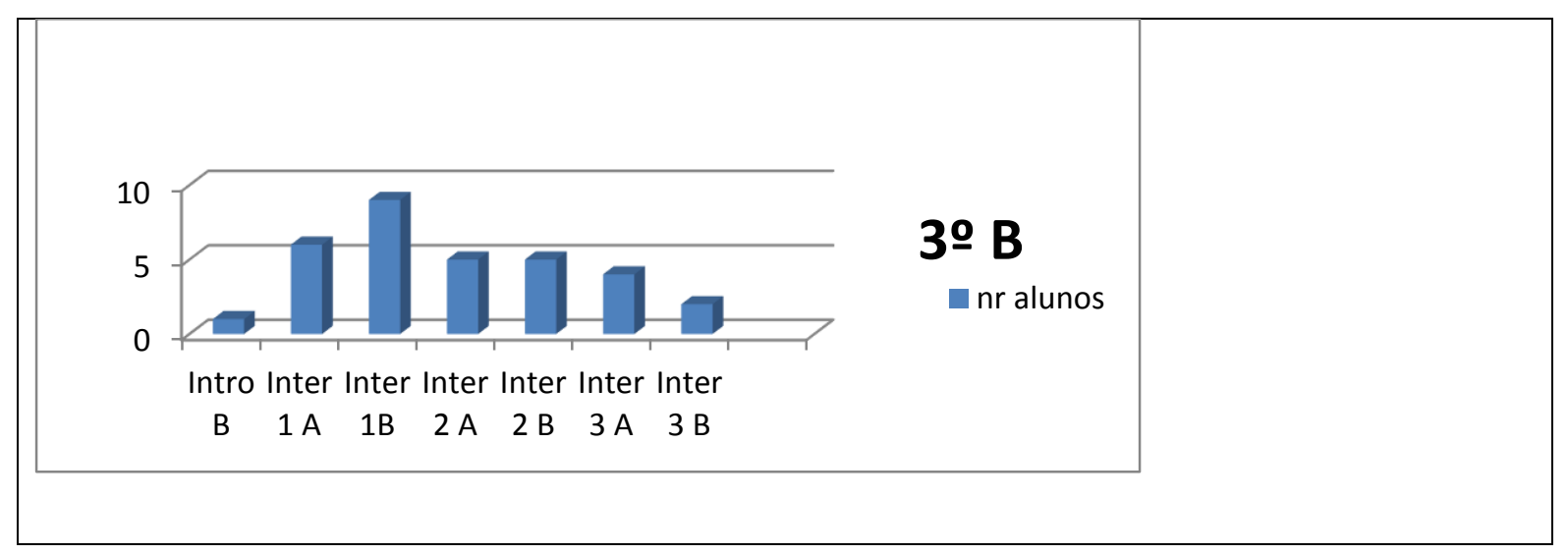

No gráfico abaixo pode-se perceber a referencia de nível.

\begin{tabular}{|c|c|c|c|c|}
\hline & & \multicolumn{3}{|c|}{ REFERÊNCIA DE LIVRO E NÍVEL } \\
\hline & & LIVRO & | NIVEL & Carga horária ideal \\
\hline & & INTROA & A1 & \\
\hline nivel & nralunos & INIRO B & A1 & \\
\hline Intro B & 1 & $\begin{array}{l}\text { NIER 1A } \\
\text { INTER 1B }\end{array}$ & $\begin{array}{l}{ }_{A 2} 2 \\
A_{2}\end{array}$ & $180 / 200$ \\
\hline Inter 1A & 6 & INTIER 2A & $A 2 / B 1$ & \\
\hline & & INTER 2B & B1 & \\
\hline Inter 1B & 9 & INTER 3 A & B1 & \\
\hline Inter 2A & 5 & PASSAGES 1 A & B2 & \\
\hline Inter 2B & 5 & PASSAGES 1 B & B2 & $500 / 600$ \\
\hline Inter 3A & 4 & PASSAGES 2 & C1 & $700 / 800$ \\
\hline Inter 3B & 2 & & & \\
\hline & & & & \\
\hline
\end{tabular}

No ensino médio integrado, 459 alunos fizeram o teste e a maior parte dos alunos se encontram no nível A2 


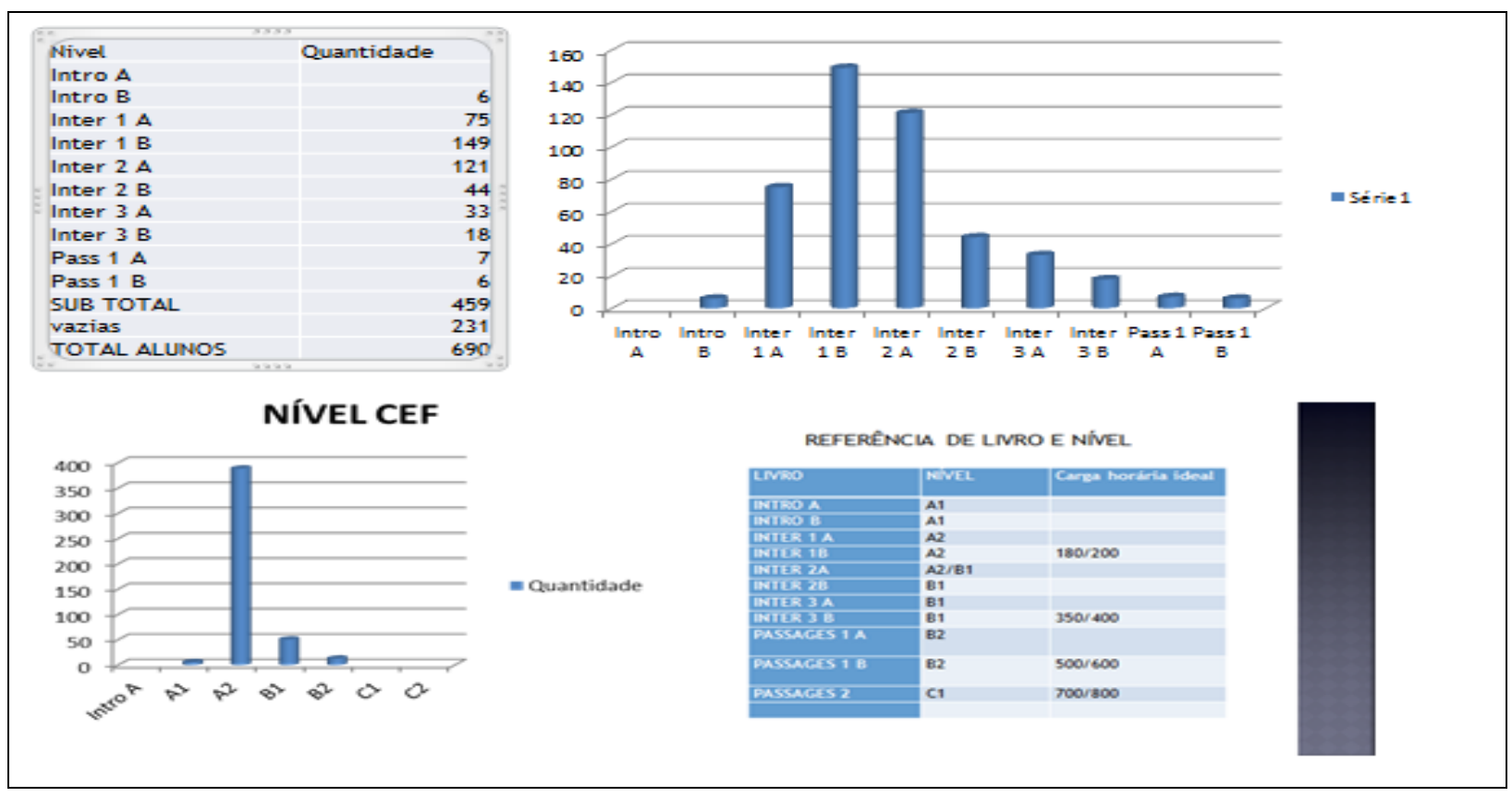

O site abaixo explica os tipos detestes de proficiência aplicados pela Universidade de Cambridge para avaliar os níveis dos alunos.

http://www.cambridgeenglish.org/exams-and-qualifications/

http://www.cambridgeenglish.org/exams-and-qualifications/preliminary-for-schools/

http://www.cambridgeenglish.org/exams-and-qualifications/key-for-schools/whats-in-theexam/

Segue um exemplo de teste oral exemplificando o que se espera de um nível B1. 


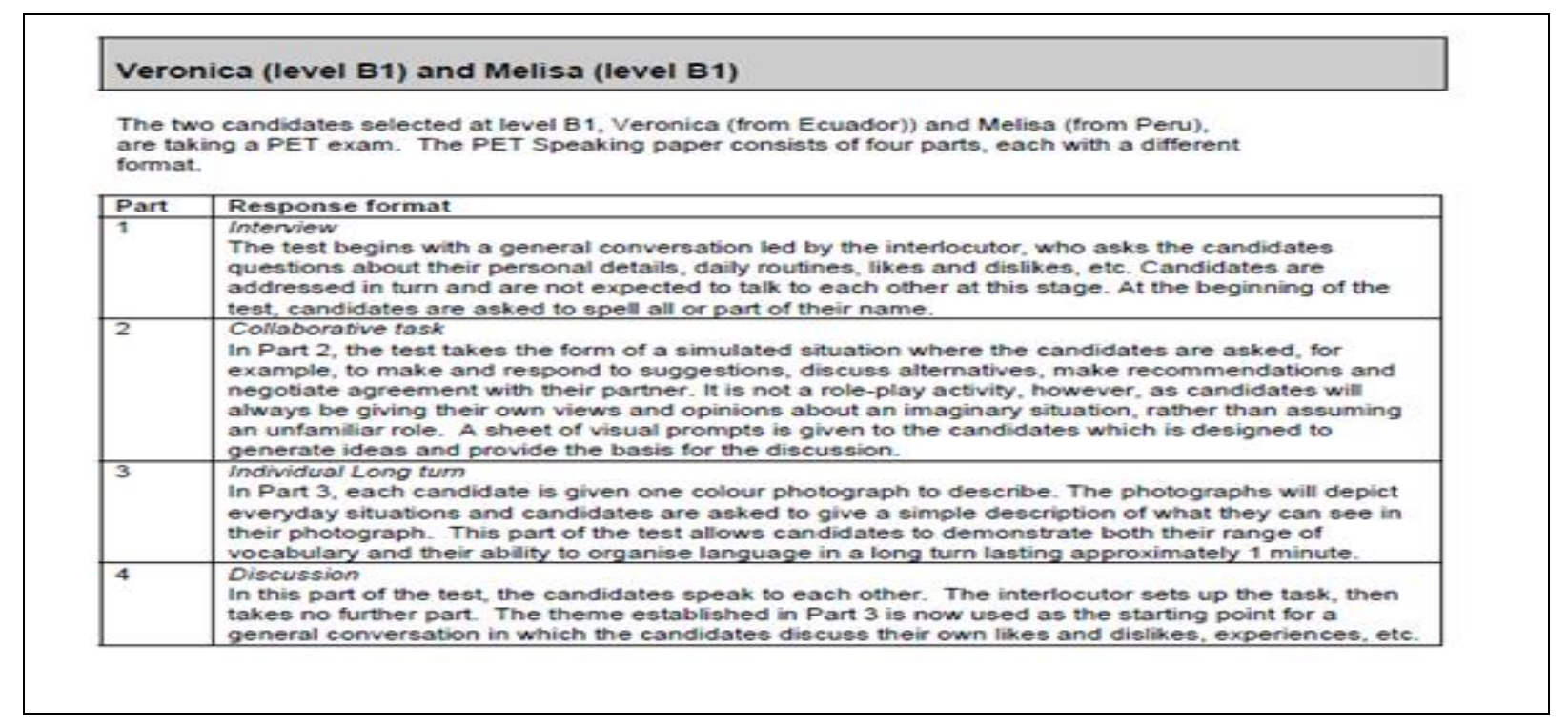

Abaixo pode-se observar Verônica Level B1.

\begin{tabular}{|c|}
\hline $\begin{array}{l}\text { Veronica: Level B1 } \\
\text { Veronica can link phrases into connected speech to produce simple but comprehensible language. She is } \\
\text { reasonably accurate in predictable situations, and so demongtrates a B1 level of performance. She does not } \\
\text { peoduce the level of detailed description or accuracy required in a B2 performance. }\end{array}$ \\
\hline 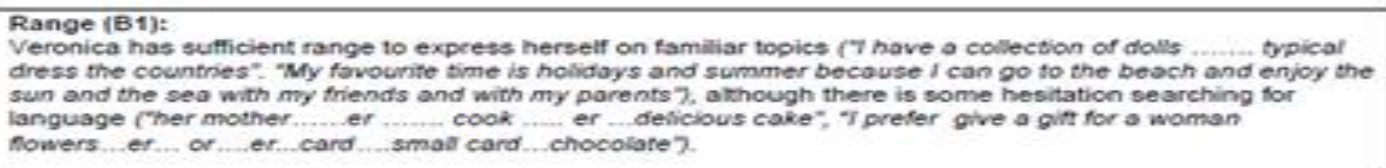 \\
\hline 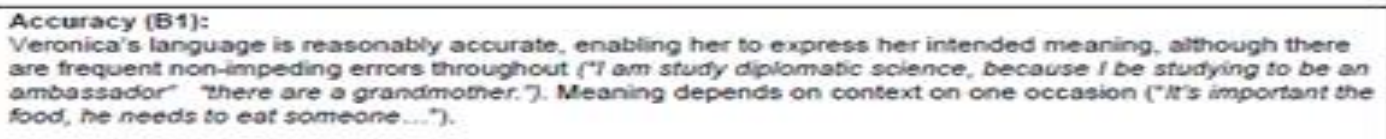 \\
\hline 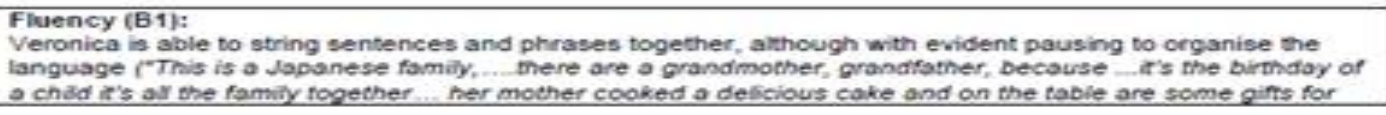 \\
\hline the birthdoy. All the famaly are very happy?. \\
\hline 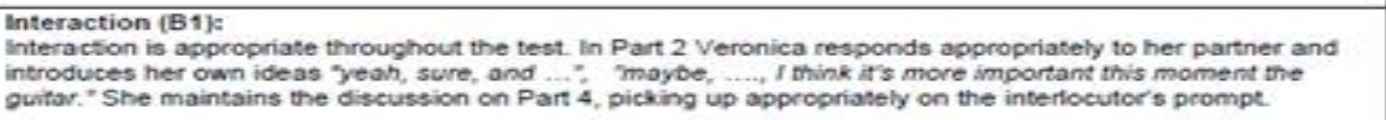 \\
\hline 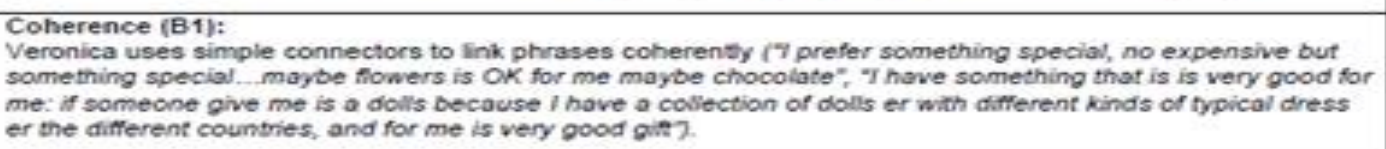 \\
\hline
\end{tabular}

Abaixo pode-se observar VMelissa Level B1. 
Melisa: Level B1

Melisa is able to keep going and produces linked phrases with a degree of accuracy in predictable situations. but with some evident pausing to organise the language. This places her in the B1 band. She produces more connected language than would be associated with an A2 performance, but lacks linguistic repertoire to produce the sustained language associated with B2.

Range (B1):

Melisa has the linguistic resources to express herself with some confidence ( 7 work in a company which export fruit and vegetable. I am quality assurance manager." There are two people. They are married. I think it the girl birthdoy and he give her a present. 2. There are frequent hesitations searching for language, however ("A gift... and some flowers ... the scene is in a kitchen and she is making the breakfast, er some fruit er a cup of tea... Or coffee, is a cup of coffee, and some cereals." "is important the, the, the .... action." because $I$ think Rome is fantastic.. is a beautiful city and you can find many ......museum.?

Accuracy (B1):

The level of accuracy is reasonable. The meaning is always clear despite frequent errors ("The country which I most visit is italy........ If you don $t$ be worry in the ... the .... with the people, you can ... use your Walkman and listen the music that you prefer or play in a little Nintendo." "And how is important relox, is important study too, and you can practice math or another science.?.

\section{Fluency (B1):}

Melisa keepe going, and produces quite extended contributions, although there is frequent pausing to organise and repair language. Her contribution at the beginning of the last part of the test demonstrates this: ("Well, I prefer receive small presents er... Iike er depend ... if is my boytriend I prefer things that when he was waiking he see, ....he saw this present and buy me. Er.. It's not important if it big or expensive, it's important the the .. the .... action .... Wh huh .... and normally for a birthday l prefer to be with ... fo meet with all the family. Gifts are not important because $I$ can buy alone... my own ..... things."?

Interaction (B1):

Although Melisa usually starts the discussions, she also responds appropriately. She supports her partner very naturally in the discussion tasks by showing she is following ("uh huh, .... Good", "oh... that's fantastic, very nice.?

\section{Considerações parciais}

Como mencionado anteriormente, o projeto está em andamento, mas vemos pelo resultado que é possível aprender a falar inglês no decorrer dos sete anos que a criança passa na escola, sem a necessidade de frequentar aulas em escolas de idiomas. Foi feito um levantamento para saber quantos destes alunos estudavam inglês em escolas de idiomas e obtivemos uma porcentagem de $10 \%$. Sendo assim, o trabalho todo está realmente sendo feito no colégio. A explicação em vídeo sobre este projeto encontra-se em:

http://www.youtube.com/watch?v=GN vc $\underline{Z U Z N k Y}$

\section{Referências Bibliográficas}

Common European Framework disponível em:

http://www.inglesnapontadalingua.com.br/ 2008/09/common-europeanframework.html. Acesso em 21/11/20137:42. Examples of speaking tests disponível em

http://www.cambridgeenglish.org/researchand-validation/fitness-for-purpose/\#B1.

Acesso em 21/11/2013 - 7:55.

Hutchinson, Tom. Project 1 and 2. Oxford University Press. Índice de Proficiência em Inglês (EF EPI) disponível em http://www.ef.com.br/epi/ Acesso em 21/11/2013 7:09

LARSEN-FREEMAN, Diane. Techniques and parinciples in language teaching.London: Oxford

UniversityPress.1986 
Memórias de aprendizagem de professores de língua inglesa disponível em: http://www.veramenezes.com/narprofessor es.htm. Acesso em 21/11/2013 - 7:05 MENEZES. Vera. A LDB e a legislação vigente sobre o ensino e a formação de professor de língua inglesa. 2003. Disponível em:

http://www.veramenezes.com/ensino.htm. Acesso em 30 out 2013.

Parâmetros curriculares nacionais: terceiro e quarto ciclos do ensino fundamental / língua estrangeira /Secretaria de Educação Fundamental. - Brasília: MEC/SEF, 1998

RICHARDS, Jack C.. Interchange Fourth Edition Intro, 1, 2, 3, CUP 2014 